\title{
Use of Palmtop Computers for Monitoring Traffic
}

\author{
M. Bíl* \& M. Bílová \& J. Martínek \\ Transport Research Centre, Czech Republic. \\ *Corresponding author: michal.bil@cdv.cz
}

\begin{abstract}
On the basis of a cycling traffic count carried out in the town of Olomouc a new method of monitoring traffic was presented based on the use of palmtop computers (PDAs). For short-term traffic counts, this method may entirely replace the commonly used paper forms. In addition, this method provides more possibilities, namely the recording of the exact time of vehicle passing and a description of the direction of vehicle movement. Almost all the software used for the preparation and processing of data is freely available, which allows all interested people to use this method.
\end{abstract}

KEY WORDS: Monitoring, traffic count, palmtop computer, PDA, cycle traffic.

\section{INTRODUCTION}

For responsible decision-making in planning the construction of new roads it is necessary to have an idea of their future traffic loads. This applies both to roads used for motor traffic and to the network of cycle paths and cycle routes. Applications for grants for the construction of cycle paths require data on the numbers of vehicles that use a given road. Likewise, it is good to know the number and demographic structure of cyclists who frequent these roads. To meet these requirements manual monitoring of cycling traffic is usually carried out. The data thus obtained can also be used to study the use of bicycles.

Cycling can be performed for the following purposes:

Commuting - regular or occasional commuting to work, school, etc. Since 2001, this kind of bicycle use has been monitored by, for example, a questionnaire survey carried out by the Czech Statistical Office. This is also the principal argument for the sake of the construction of cycle paths. Their separation from motor traffic should improve road traffic safety (KONKIN et al., 2006; RICHTER et al, 2007).

Recreation - concerns occasional rides, usually on terrain which is different from that used when commuting rides are taken. Surveys of recreational cycling are carried out especially in major tourist areas, where it belongs to the category of "monitoring of visitors” (e.g., COPE et al., 2000). The initiative of the National Park České Švýcarsko, where, since 2008, the frequency of cycling has been monitored with the use of automatic sensors, is an example of cycling traffic counts in Czech natural areas (verbal information). But cycle tourists also like to use the backbone network of cycle routes of the systems EuroVelo and North Sea Cycle Route, (e.g. LUMSDON et al, 2004.). 
Sport or training - the terrain, type of a bicycle and ride intensity differ according to the kind of sport practiced.

The monitoring of cycling should always be organized in a way which allows for distinguishing between all kinds of bicycle use. For example, it is desirable to monitor commuting to work before the anticipated beginning of working hours, i.e., in the morning. In the afternoons there is already a likelihood of data "contamination" by recreational cycling traffic.

According to the objectives and the method used it is possible to divide traffic monitoring generally into the two following categories:

1. the collection of quantitative data, particularly on the frequency of a given vehicle type, which is usually called the traffic count,

2. the collection of socioeconomic and demographic parameters of drivers and passengers, which is usually performed in the form of questionnaire surveys or interviews.

A traffic count is either carried out manually or with the use of automatic, mostly electronic, counters. These counters are useful if it is necessary to count for a relatively long period, e.g., one week and longer. There are many technologies that can be used for the detection of cyclists (pressure sensors, infrared detectors, induction loops, etc.). But the use of most available methods is considerably limited in that they can not be used to determine some other characteristics of cyclists, such as the age, sex, clothing, helmet, etc. Limitations of most sensors include the fact that they can not distinguish a cyclist from a pedestrian, or a cyclist from a car. Though it is possible to connect a movement sensor with a camera and make an image analysis, the obtained results - unlike those for the detection of motor vehicles - have so far been unsatisfactory for the monitoring of cycling. There have been promising attempts to distinguish cyclists and motorcyclists (MESSELODI et al., 2007), however similarly to the video detection of cyclists and pedestrians (HEIKKILA and SILVÉN, 2004), they do not yet have commercial applications.

For occasional campaign counts where it is required to find out the current traffic situation at several places, a counting staff is employed. They enter data, usually sums for a given type of vehicle, into pre-prepared paper forms. Regular traffic counts organized by the Road and Motorway Directorate of the Czech Republic (RSD) are carried out this way. Shortcomings of this process involve the necessity to subsequently retype the data into a PC, and the potential errors introduced thereby. Another limitation consists of the inability to record the exact time of passing of a given vehicle. The sums relate to the day of counting.

The process of data collection that we present fills the gap between the automatic data collection (traffic count) on the one hand, and the questionnaire survey on the other hand. It has a potential to streamline the work of the counting staff by introducing electronic forms in a PDA environment, and an intuitive pseudo-map interface directly on the device display.

\section{METHODS}

To entirely replace paper forms and enhance the monitoring by introducing other potentialities that involve, in particular, recording the exact time of a vehicle passing, we therefore used palmtops - PDAs (Personal Digital Assistant). The advantages of PDAs when compared to paper forms consist particularly of the following: 
a. Once downloaded to a PC the data collected is immediately available, so there is no need to retype it, which is often a source of errors.

b. Nowadays, PDA technology is easily accessible. It is not financially demanding and can be used for everyday work as an organizer, etc.

c. It is possible to adjust the appearance of counting forms for a particular event and the environment to match the situation at a particular place (unlike Traffic Data Collectors, which represent mobile devices designed as a hardware module for traffic counts at intersections - e.g., the Jamar technology).

d. A great advantage of PDAs lies in the possibility to record the time of a vehicle passing, making these data applicable for further use. This constitutes an important connection between the conventional counting and filling in forms.

For traffic counts only such a device can be used that can operate in a battery mode for the time needed to collect data, which is usually several hours. If there's bad weather it is good to have the possibility to use a rain protective cover.

The preparation of the form for work is as follows. The PC needs to have XS Designer software installed, which is the environment for the form production. A single form may contain more pages, which may include different controls such as: descriptions, text boxes, check boxes, option buttons, combo boxes, date and time, pictures and buttons. An action can be set for each control, either by selecting from predefined controls, or by creating customized ones in the programming languages JScript or VBScript. In addition, SQL inquiries can be defined and assigned to controls of the form. Operation of the form can be tested under condition that XSForms software is installed on PDA and the PDA is connected to a computer.

We had 10 pieces of PDA, type Fujitsu SIEMENS FSC Pocket LOOX N560 with an active touch screen at our disposal. During the preparatory phase we prepared plans of given counting sites in the programme PhotoFiltre. These sites were usually located at intersections, where each of the four directions was assigned a letter A - D (Figure 1). The software PhotoFiltre is a simple graphic editor for creating and editing raster images. Among other things, it also supports working with layers. To create and transfer the prepared form from PC to PDA, we used the programme XSDesigner. In PDA, the form can be opened in the programme XSForms which cooperates with XSDesigner.

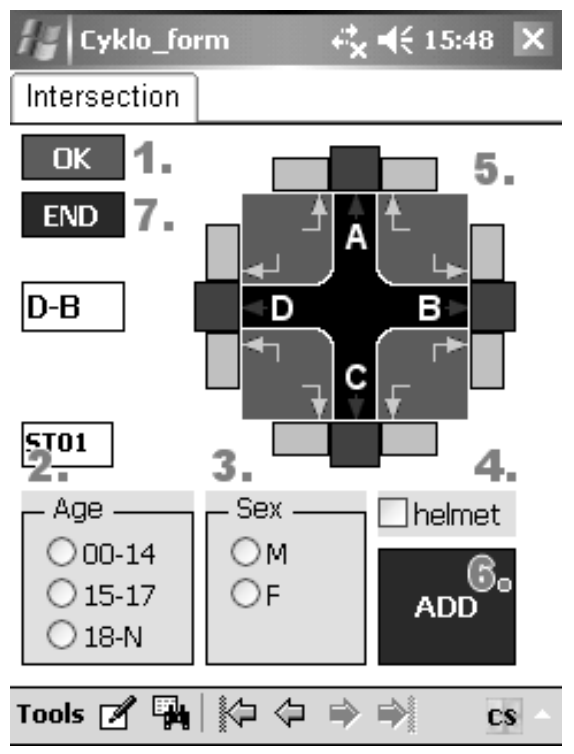

Figure 1: Example of a form in PDA. 
Prior to each measurement, the counting staff met together to synchronize the time in their PDAs with an accuracy in seconds. Then everyone went to their stands and at a given time, in our case at 5:30, started the traffic count by pressing the "Start" button on his/her PDA display (see Figure 1).

The passing of the first cyclists through the intersection was then awaited. Given that the counting sites had been chosen to provide the staff with a good view, it was possible to estimate the cyclist's age, sex, and the presence of a protective helmet prior to his/her approach to a counting site. Once the direction had been evident in which the cyclist would leave the intersection, his/her passage route was recorded (point 5 in Figure 1). When passing straight through the intersection, the middle of the three buttons (red) was pressed for the direction in which the cyclist had left the intersection. If he/she had ridden, for example, from D to B, the middle button was pressed for B. If the cyclist had turned, the outer buttons (yellow) were pressed. For example, if he/she had turned from D to A, the left button was pressed for A. This intuitive interface with active elements on the display makes the work very quick.

By pressing the "Add” button the exact time of cyclist's passing was entered. At the end of the count at 8:00 am the "End" button was pressed to finish the data collection and close the programme. If, within the counting process, the staff had become aware of some errors made, it was still possible to correct them at the count site. The form allows moving between records (arrows in the lower part of the form).

In the course of the count the staff were in connection with the coordinator of the action via mobile phones. It was therefore possible to operatively aid the staff if the count was jeopardized by a drop of battery voltage on some particular PDA. To solve such situations, the traffic count coordinator was equipped with a mobile power pack - APC Mobile Power Pack UPB10.

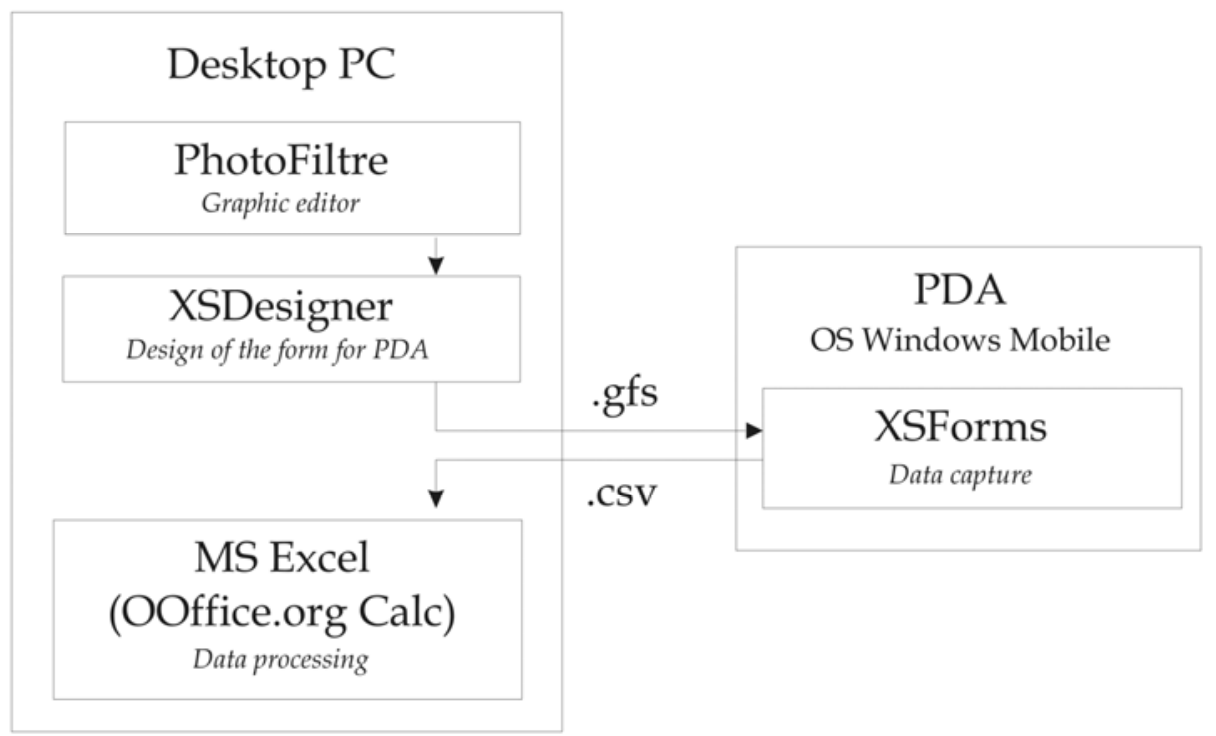

Figure 2: Diagram of the procedure of data preparation and processing.

After finishing the traffic count the form was directly exported in the application XSForms to a new file in CSV format. This file was subsequently transferred to a desktop computer using the software Microsoft ActiveSync which is used for synchronizing mobile devices with the system Windows Mobile. Using a PC data were imported into the programme MS Excel for analysis of the volumes of cyclists riding in individual directions and at certain times. This procedure is schematically illustrated in Figure 2. 
Although the whole working process is very efficient, it is necessary to reckon with some constraints, especially for older hardware. These constraints may particularly involve the worse screen readability of PDAs outdoors, lower battery life (usually less than 3 hours depending on the intensity of the display backlight), and the capability of PDAs to work at lower temperatures.

Regarding the work coordination it is necessary to not forget to synchronize the time before the start of a count.

\section{APPLICATION}

We initially applied this method to carry out a cycling traffic count in the town of Olomouc. Due to the fact that the centre of the town is a historical reserve with the original historic pavement, it is not convenient for this type of transport. In its vicinity, however, there is an almost perfectly coherent system of urban parks which follow the foreground of a former fortress. Also the topography of the town favours the use of bicycles.

The network of cycle paths in Olomouc has been built and expanded since 1994. The total length of available cycle paths within the territory of the town is $23 \mathrm{~km}$, but cyclists also frequently use the network of streets that they share with motor vehicles (TÁZLAR, 2007). We focused our research only on the vicinity of the centre where we identified 10 counting sites. We placed an emphasis on detecting the main routes for commuting to work.

The cycling traffic count was carried out in March 2009 within two weeks (1012 March, and 17 - 19 March) on Tuesdays to Thursdays from 5:30 am to 8:00 am in order to cover the middle of a working week (we assume that on Mondays and Fridays, commutation may have a different pattern), and morning commutes to work and schools. The cycling traffic count was carried out off the main cycling season because we focused mainly on those cyclists who commute regularly. In our opinion the seasonal cyclists would not commute during this time, as well as those cyclists for whom cycling is a leisure activity. We prepared a data sample based on an exemplary day, Wednesday, 18 March, in order to provide an introduction to the traffic count method with the use of PDA.

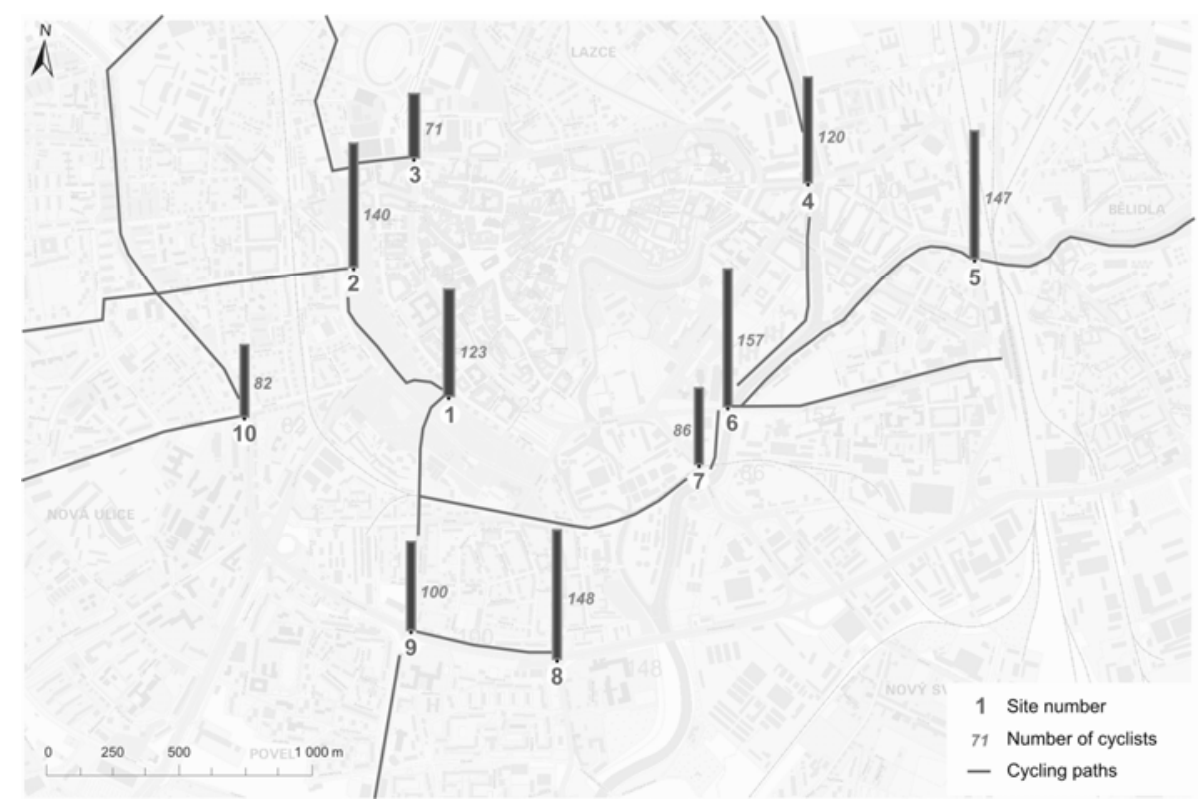

Figure 3: Number of cyclists passing traffic count sites and their localization in the selected part of the town of Olomouc. 
Figure 3 shows the centre of the town of Olomouc. The dark line marks the constructed parts of cycle paths that are separated from motorised traffic. Since the network is still incomplete, the cyclists need to use roads for motorised traffic, too. The height of columns and numbers next to them show the number of cyclists who passed a traffic count site on that day between 5.30 a.m. and 8.00 a.m., regardless of the direction of riding.

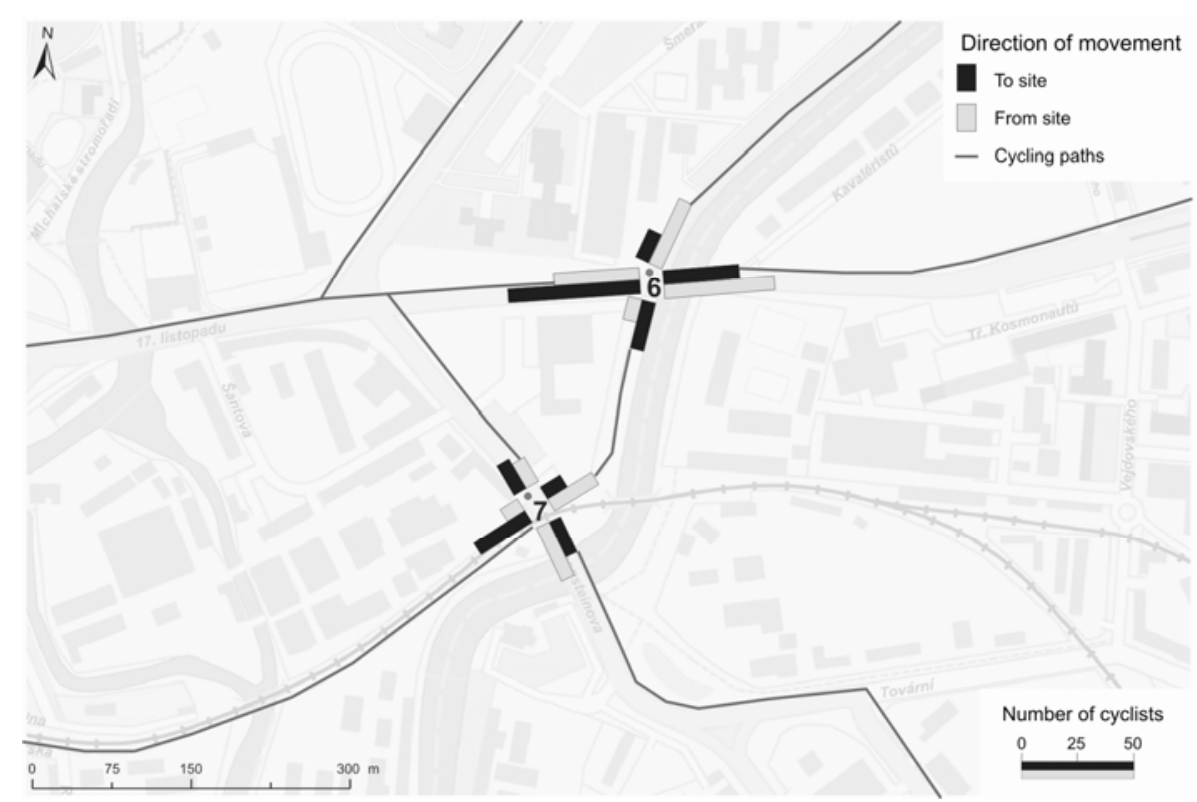

Figure 4: Direction of movement of cyclists within stations 6 and 7.

Figure 4, traffic count sites of 6 and 7, shows the visualized results in GIS. The displayed digital form has the advantage of capturing the direction of movement of cyclists. Individual columns vary in colour, based on the fact whether cyclists arrive at a traffic count site (black) or depart from a traffic count site (grey).

Table 1:Recorded cyclists having passed traffic count sites 6 and 7 at times $5.30-6.00$ on 18 March 2009.

\begin{tabular}{|c|c|c|c|c|c|c|}
\hline $\begin{array}{c}\text { Direction } \\
\text { from no. }\end{array}$ & $\begin{array}{c}\text { Departure } \\
\text { (hh:mm:ss) }\end{array}$ & $\begin{array}{c}\text { Arrival } \\
\text { (hh:mm:ss) }\end{array}$ & $\begin{array}{c}\text { Speed } \\
(\mathrm{km} / \mathrm{h})\end{array}$ & Sex & $\begin{array}{c}\text { Helmet } \\
(\mathrm{Y} / \mathrm{N})\end{array}$ & $\begin{array}{c}\text { Age } \\
\text { group }\end{array}$ \\
\hline 6 & $5: 35: 20$ & $5: 36: 05$ & 20 & $\mathrm{M}$ & $\mathrm{Y}$ & $>18$ \\
\hline 7 & $5: 37: 15$ & $5: 37: 58$ & 21 & $\mathrm{M}$ & $\mathrm{N}$ & $>18$ \\
\hline 7 & $5: 38: 00$ & $5: 38: 52$ & 17 & $\mathrm{M}$ & $\mathrm{N}$ & $>18$ \\
\hline 6 & $5: 42: 34$ & $5: 43: 13$ & 23 & $\mathrm{~F}$ & $\mathrm{~N}$ & $>18$ \\
\hline 7 & $5: 45: 07$ & $5: 45: 56$ & 18 & $\mathrm{M}$ & $\mathrm{N}$ & $>18$ \\
\hline 6 & $5: 48: 41$ & $5: 49: 11$ & 30 & $\mathrm{~F}$ & $\mathrm{~N}$ & $>18$ \\
\hline 6 & $5: 53: 17$ & $5: 53: 56$ & 23 & $\mathrm{M}$ & $\mathrm{Y}$ & $>18$ \\
\hline 7 & $5: 54: 55$ & $5: 55: 33$ & 24 & $\mathrm{M}$ & $\mathrm{Y}$ & $>18$ \\
\hline 7 & $5: 54: 01$ & $5: 54: 57$ & 16 & $\mathrm{M}$ & $\mathrm{Y}$ & $>18$ \\
\hline 7 & $5: 58: 10$ & $5: 58: 58$ & 19 & $\mathrm{M}$ & $\mathrm{Y}$ & $>18$ \\
\hline
\end{tabular}


Table 1 shows the outcome of counting in the table processor application in order to determine the average speed of a cyclist. In the morning between $5.30 \mathrm{a} . \mathrm{m}$. and $6.00 \mathrm{a}$.m. six cyclists arrived at site 6 (direction from site 7) out of the total daily number of 27 cyclists arriving from that direction, see Figure 4. Four cyclists (out of 13 arriving, or 28 departing cyclists) arrived at site 7 (direction from site 6).

\section{DISCUSSION AND CONCLUSION}

The method we propose can replace conventional traffic volume counts carried out with the use of paper forms. In addition, it also allows for the recording of exact times of passing of selected road users (in this case - a cyclist). Similarly to situations where a motion sensor is installed in the site of a traffic count profile, we obtain information on the volumes of cycling traffic at arbitrary time steps, but also further data, e.g., safety helmet wearing, age group, sex, reflective clothes, etc. Monitoring at intersections allows for the recording of information about travel directions.

We can only benefit from the advantages of the use of pocket PCs - PDAs compared with the use of paper forms if these devices meet the requirement of several hours' operation in the field. The whole working process and the process of data processing uses software which is either freely available or widely used and can be easily substituted (e.g., MS Excel for OpenOffice Calc).

Although there are sophisticated devices for traffic counting on junctions (e.g. Traffic Data Collector by Jamar Technologies, Inc), our introduced solution is flexible thanks to the fact that a customized form design (junction) can be prepared in advance and controls deployed according to the needs and purpose of an action.

\section{ACKNOWLEDGMENT}

For their cooperation in the cycling traffic count carried out many thanks to the employees and students of the Department of Geoinformatics of the Faculty of Science of the Palacký University in Olomouc. Our contribution was supported by the project of the Ministry of Transport No. MD04499457501.

\section{REFERENCES:}

Cope, A. - Doxford, D. - Probert, C. Monitoring visitors to UK countryside resources. The approaches of land and recreation resource management organisations to visitor monitoring. Land Use Policy, 17, (2000) 59-66.

HeikkilA, J. - Silvén, O. A real-time system for monitoring of cyclists and pedestrians. Image and Vision Computing 22 (2004) 563-570.

Konkin, D.E. - Garraway, N. - Hameed, S.M. - Brown, D.R. - Granger, R. - Wheeler, S. Simons, R.K. Population-based analysis of severe injuries from non-motorized wheeled vehicles. The American Journal of Surgery, 191, 615-618, 2006.

Lumsdon, L. - Downward, P. - Cope, A. Monitoring of cycle tourism on long distance trails: the North Sea Cycle Route. Journal of Transport Geography 12 (2004) 13-22. 
Messelodi, S. - Modena, C.M. - Cattoni, G. Vision-based bicycle/motorcycle classification. Pattern Recognition Letters 28 (2007) 1719-1726.

Richter, M. - Otte, D. - Haasper, C. - Knobloch, K. - Probst, C. - Westhoff, J. Sommer, K. - Krettek, C. The Current Injury Situation of Bicyclists-A Medical and Technical Crash Analysis, J Trauma. May;62(5):1118-22, 2007.

Tázlar, J. Mapping of cyclist infrastructure on the territory of the town of Olomouc, 2007. 58p. Diploma thesis on the Faculty of Science of the Palacký University in Olomouc, Department of Geoinformatics. Supervisor of diploma thesis - Michal Bíl. 